\title{
Paper
}

\section{Parametric study for directional stability of tumbling plates by wind tunnel testing}

\author{
Mitsuo Ishiguro* ${ }^{*} \quad$ Member, $\quad$ Yoshiaki MiYaKe ${ }^{\dagger}$ Non-member
}

(Received October 26, 2021, revised January 7, 2022)

\begin{abstract}
The tumbling phenomenon is considered to be an important phenomenon for aircraft flight safety because of the possibility of falling objects from the aircraft reaching far away or for knowing the behavior of falling roof tiles blown off by strong winds. Therefore, many tests to measure the force act on the tumbling plate in wind tunnel have been conducted. The authors have been studying the directional stability of the tumbling plate [9], which has a great effect on the reach of falling objects, and have proposed a non-contact measurement method wind tunnel test method from the viewpoint of dynamic balance which can acquire the force without force sensors [12]. Here, we call "towing wind tunnel test method". In this paper, for the evaluation of the directional stability of the tumbling plate, we parametrically changed the planar configuration of the tumbling flat plate and measured the acting force in wind tunnel using this towing wind tunnel test method. As a result, the relationship between the shape of the tumbling plate and the conditions for directional instability was clarified.
\end{abstract}

Keywords: Tumbling, Wind tunnel, Directional stability, Stereo Imaging,

\section{Introduction}

When an object falls in a fluid, continuous rotation by the action of fluid force is called autorotation. Autorotation performs various modes of motion by performing complicated motions according to the dimensions of a falling object, velocity, moment of inertia, aerodynamic characteristics, etc. As motion modes, tumbling, flat spin, corning, etc., are known. Among the autorotation, the drop direction and the rotation axis are vertical is called tumbling.

Tumbling is known as natural phenomena such as leaves of trees and roof tiles blown away by typhoons, and important phenomenon in aerospace engineering and meteorology as well as scattering region estimation of satellite/rocket fragments damaged during atmospheric reentry and objects dropped from aircraft at random. There are many studies for analysis of the tumbling motion by making full use of analysis methods [1] [3] [5], or establishing a mathematical model [4]. And also many studies have been conducted due to clarify the tumbling phenomenon and how the speed and glide ratio of the falling object change depending on conditions such as Reynolds number and aspect ratio [6] [7], and some empirical equations have been proposed [8].

Although most of the studies so far assume a motion in a two-dimensional plane perpendicular to the ground. Also, as a measuring method by the free fall, it is difficult to identify the aerodynamic force under specific attitude angle conditions especially associated with the lateral motion from the three-dimensional coordinates. However, the above de-

\footnotetext{
* Corresponding: my-ishiguro@ nifty.com

$\dagger$ Aichi Institute of Technology

1247 Yachikusa, Yakusa-cho, Toyota, Aichi, Japan 470-0392
}

scribed problem like scattering region is greatly affected by the directional stability of the falling object. This is because when the falling object starts to turn, the area to reach is limited to the vicinity of the position where the falling object started to fall. Authors have focused on this directional stability of the tumbling plate and studied the directional stability for typical configurations [9]. Also, authors have proposed a new towing wind tunnel test method of the tumbling plate [12].

In this study, the effect of the plane configuration of the tumbling plate, which is parametrically changed, on the directional stability is examined by developed towing wind tunnel test method and measuring the periodic aerodynamic force during tumbling.

\section{Force Conversion Method by Position Measurement}

Here, we overview the towing wind tunnel test method for the tumbling plate proposed by the authors [12].

Consider that both ends of a tumbling flat plate rotational axis to be held by two strings from the front in a wind tunnel (Fig. 1). The string and the rotational axis of the tumbling plate are connected so that it can rotate freely, and the other end of the strings are supported from the outside of the wind tunnel. For simplicity, the aerodynamic force or gravity acting on the string itself shall be negligible (this assumption will be discussed later). Although it is known that the aerodynamic force applied along the rotation cycle changes periodically in tumbling motion [7], we treat the force that averages the change in one cycle in this study.

Now, assume that a tumbling plate in the uniform flow is held by right and left strings pulling to different directions relative to the plate (Fig. 2). At this time, the tumbling plate 


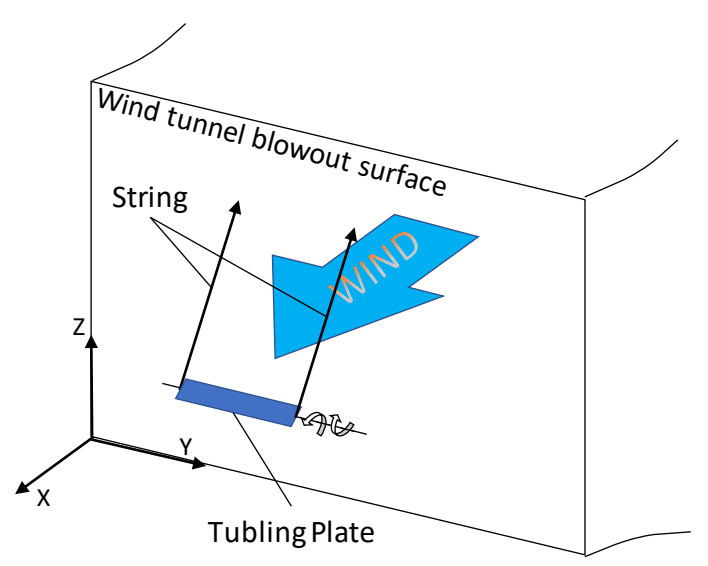

Figure 1: Wind tunnel test image.

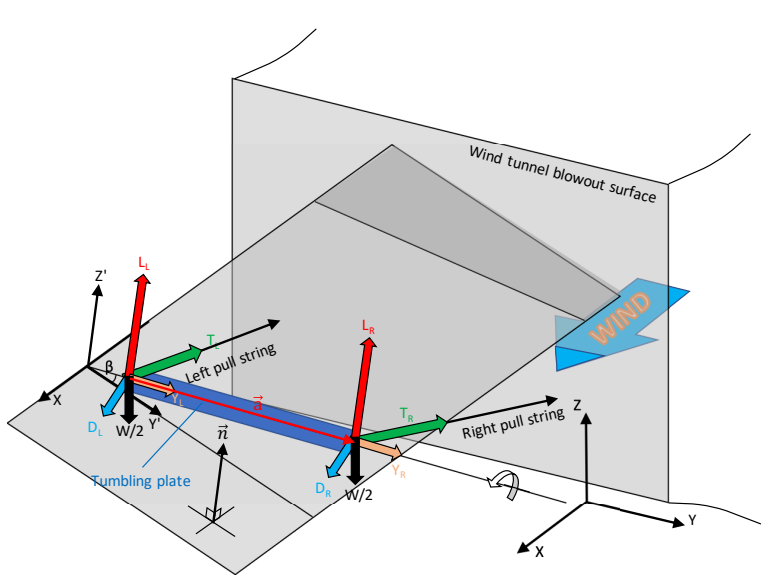

Figure 2: Foces in non-symmetrical case in wind tunnel.

is rotating in the direction of the white arrow at certain angle with respect to the uniform flow and is generating lift in the upward direction. In the figure, the $X Y Z$ axis is the axis system fixed to the wind tunnel, and the wind blows in the $X$ direction.

Now, take the vector $\vec{a}$ from the left end point to the right end point of the tumbling plate in the direction of the rotational axis, and set the unit vector as $\overrightarrow{e a}$. Consider a plane containing $\vec{\alpha}$ and the $X$ axis and set the unit vector of its normal vector $\vec{n}$ and take the $Z^{\prime}$ axis in same direction. We can set $X Y^{\prime} Z^{\prime}$ axis system and the sideslip angle $\beta$ of the tumbling plate is an angle formed by $\vec{a}$ and $Y^{\prime}$ axis. We define the unit vector in the $X, Y^{\prime}$ direction as $\overrightarrow{e x}, \overrightarrow{e y}$, unit vector in $Z$ direction as $\overrightarrow{e z}$. Unit vector in $Z^{\prime}$ direction is unified by $\vec{n}$.

The force acting on the tumbling plate can be divided into the forces acting on the right and left ends. The Lift force is divided into $L_{L}$ and $L_{R}$ acting in the $Z^{\prime}$ direction, the drag force is divided into $D_{L}$ and $D_{R}$ acting in the normal direction of the plane including the $\vec{a}$ and $Z^{\prime}$ axis, side force is divided into $Y_{L}$ and $Y_{R}$ acting in the direction of the rotational axis. The weight of the tumbling plate is expressed as $W$ is divided into $W / 2$ acting equally on the right and left sides in the $Z$ direction. For the tension, the tension applied to the left and right strings are expressed as $T_{L}$ and $T_{R}$, and the unit vector in the pulling direction of each of them is expressed as $\overrightarrow{e T_{L}}, \overrightarrow{e T_{R}}$.

The drag, side force and tension are expressed as follows using the unit vector.

$\begin{array}{ll}\text { Drag: } & D_{L} \overrightarrow{e D}, D_{R} \overrightarrow{e D} \\ \text { Side force: } & Y_{L} \overrightarrow{e a}, Y_{R} \overrightarrow{e a}\end{array}$

String Tension: $T_{L} \overrightarrow{e T_{L}}, T_{R} \overrightarrow{e T_{R}}$

By measuring the three-dimensional position of the end point of the tumbling plate and the holding point of the string $\vec{a}, \overrightarrow{e a}$, and $\overrightarrow{e T_{L}}, \overrightarrow{e T_{R}}$ are obtained. $\vec{n}$ is obtained from the cross product of $\overrightarrow{e x}$ and $\overrightarrow{e a}$.

$$
\vec{n}=\frac{\overrightarrow{e x} \times \overrightarrow{e a}}{|\overrightarrow{e x} \times \overrightarrow{e a}|}
$$

And $\overrightarrow{e y}$ is,

$$
\overrightarrow{e y}=\frac{\vec{n} \times \overrightarrow{e x}}{|\vec{n} \times \overrightarrow{e x}|}
$$

Unit vector in the direction of drag force is,

$$
\overrightarrow{e D}=\frac{\overrightarrow{e a} \times \vec{n}}{|\overrightarrow{e a} \times \vec{n}|}
$$

The tumbling plate can turn reverse direction. So, here assume that the rotational direction of the tumbling plate is reversed to generate a down force. At this time, the tumbling plate is located on a plane different from the $X Y^{\prime}$ plane at the time of generating the lift force. For simplicity, assume that the pulling direction of the string is adjusted and the sideslip angle in the plane in which the tumbling plate exists is set to the value of sideslip angle $\beta$. (In the wind tunnel test, some cases in which $\beta$ was slightly changed were carried out, and the same $\beta$ value was obtained by interpolating the results.)

Now, define the position at the time of generating the lift force is referred to as "upper" and at the time of generating the downforce is referred to as "lower", and express them to add the suffix of "u" and "l" respectively (use lowercase letters to distinguish between left and right suffixes $L$ and $R)$.

For each direction $X, Y^{\prime}, Z^{\prime}$ the following equation of balance holds.

upper,

$$
\begin{gathered}
D_{L} \overrightarrow{e D_{u}} \cdot \overrightarrow{e x}+Y_{L u} \overrightarrow{e a_{u}} \cdot \overrightarrow{e x}+T_{L u} \overrightarrow{e T_{L u}} \cdot \overrightarrow{e x}=0 \\
D_{L} \overrightarrow{e D_{u}} \cdot \overrightarrow{e y_{u}}+Y_{L u} \overrightarrow{e a_{u}} \cdot \overrightarrow{e y_{u}}-\frac{W}{2} \overrightarrow{e z} \cdot \overrightarrow{e y_{u}}+T_{L u} \overrightarrow{e T_{L u}} \cdot \overrightarrow{e y_{u}}=0 \\
L_{L}-\frac{W}{2} \overrightarrow{e z} \cdot \overrightarrow{n_{U}}+T_{L u} \overrightarrow{e T_{L u}} \cdot \overrightarrow{n_{u}}=0 \\
D_{R} \overrightarrow{e D_{u}} \cdot \overrightarrow{e x}+Y_{R u} \overrightarrow{e a_{u}} \cdot \overrightarrow{e x}+T_{R u} \overrightarrow{e T_{R u}} \cdot \overrightarrow{e x}=0 \\
D_{R} \overrightarrow{e D_{u}} \cdot \overrightarrow{e y_{u}}+Y_{R u} \overrightarrow{e a_{u}} \cdot \overrightarrow{e y_{u}}-\frac{W}{2} \overrightarrow{e z} \cdot \overrightarrow{e y_{u}}+T_{R u} \overrightarrow{e T R_{u}} \cdot \overrightarrow{e y_{u}}=0 \\
L_{R}-\frac{W}{2} \overrightarrow{e z} \cdot \overrightarrow{n_{u}}+T_{R u} \overrightarrow{e T_{R u}} \cdot \overrightarrow{n_{u}}=0
\end{gathered}
$$


lower,

$$
\begin{aligned}
& D_{L} \overrightarrow{e D_{l}} \cdot \overrightarrow{e x}+Y_{L l} \overrightarrow{e a_{L}} \cdot \overrightarrow{e x}+T_{L l} \overrightarrow{e T_{L l}} \cdot \overrightarrow{e x}=0 \\
& D_{L} \overrightarrow{e D_{l}} \cdot \overrightarrow{e y_{l}}+Y_{L l} \overrightarrow{e a_{l}} \cdot \overrightarrow{e y_{l}}-\frac{W}{2} \overrightarrow{e z} \cdot \overrightarrow{e y_{l}}+T_{L l} \overrightarrow{e T_{L l}} \cdot \overrightarrow{e y_{l}}=0 \\
& -L_{L}-\frac{W}{2} \overrightarrow{e z} \cdot \overrightarrow{n_{l}}+T_{L l} \overrightarrow{e T_{L l}} \cdot \overrightarrow{n_{l}}=0 \\
& D_{R} \overrightarrow{e D_{l}} \cdot \overrightarrow{e x}+Y_{R l} \overrightarrow{e a_{l}} \cdot \overrightarrow{e x}+T_{R l} \overrightarrow{e T_{R l}} \cdot \overrightarrow{e x}=0 \\
& D_{R} \overrightarrow{e D_{l}} \cdot \overrightarrow{e y_{l}}+Y_{R l} \overrightarrow{e a_{l}} \cdot \overrightarrow{e y_{l}}-\frac{W}{2} \overrightarrow{e z} \cdot \overrightarrow{e y_{l}}+T_{R l} \overrightarrow{e T_{R l}} \cdot \overrightarrow{e y_{l}}=0 \\
& -L_{R}-\frac{W}{2} \overrightarrow{e z} \cdot \overrightarrow{n_{l}}+T_{R l} \overrightarrow{T_{R l}} \cdot \overrightarrow{n_{l}}=0
\end{aligned}
$$

Since the unknowns in Eq. 4 to Eq. 15 are the following 12 scalars, all of them can be solved as simultaneous linear equations.

$D_{L}, D_{R}, L_{L}, L_{R}, Y_{L u}, Y_{L l}, Y_{R u}, Y_{R l}, T_{L u}, T_{L l}, T_{R u}, T_{R l}$

In these equations, it is a condition that $\beta$ matches between the upper position and lower position, so the coefficients related to the attitude angle of the tumbling plate (ex. $\left.\overrightarrow{e D_{u}} \cdot \overrightarrow{e x}, \overrightarrow{e a_{u}} \cdot \overrightarrow{e x}, \overrightarrow{e D_{u}} \cdot \overrightarrow{e y_{u}}, \overrightarrow{e a_{u}} \cdot \overrightarrow{e y_{u}}, e t c.\right)$, are obtained when the sideslip angle $\beta$ is determined.

The lift $L$, the drag $D$, the lateral force $Y$, the rolling moment $l$ and the yawing moment $n$ of the tumbling plate are calculated in the following order.

$$
\begin{aligned}
& L=L_{L}+L_{R} \\
& D=D_{L}+D_{R} \\
& Y=Y_{L u}+Y_{R u}, Y_{L l}+Y_{R l} \\
& l=\left(L_{L}-L_{R}\right) \cdot b / 2 \\
& n=\left(D_{R}-D_{L}\right) \cdot b / 2
\end{aligned}
$$

where $b$ is the lateral width of the shaft and the sideslip angle $\beta$ is calculated according to the definition of dot product as follows,

$$
\beta=\cos ^{-1}(\overrightarrow{e a} \cdot \overrightarrow{e x})-\frac{\pi}{2}
$$

\section{Preliminary Free Fall Test}

The authors have pointed out that the directional stability changes depending on the configuration of the tumbling plate by the free fall test [9]. The purpose of this study is to consider the stable conditions from the viewpoint of the force measured by the wind tunnel test. However, it is known that the directional stability of the tumbling plate depends on various conditions such as its size, thickness, mass, and moment of inertia, even in the same planar model. Therefore, prior to the wind tunnel test, free fall tests were conducted using the models with the same specification (without shaft) used for the wind tunnel test written in 4.2. This chapter gives an overview.

Test apparatus is shown in Fig. 3, which is same as [9].

The trajectory of falling model is acquired by using stereoscopic technique same as written in 4.4.

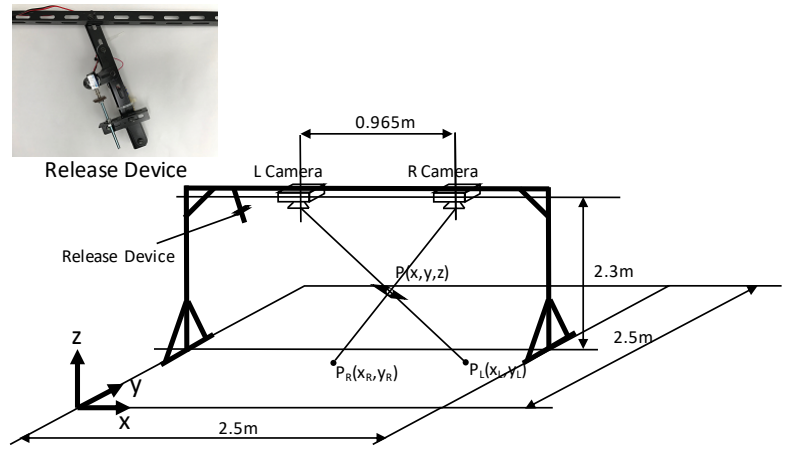

Figure 3: Experimental Apparatus.

Table 1: Percentage of Straight Trajectory [11].

\begin{tabular}{cc}
\hline Confuguration & Percentage of Straight Trajectory(\%) \\
\hline I & 100 \\
II & 100 \\
III & 20 \\
IV & 15 \\
V & 15 \\
VI & 0 \\
\hline
\end{tabular}

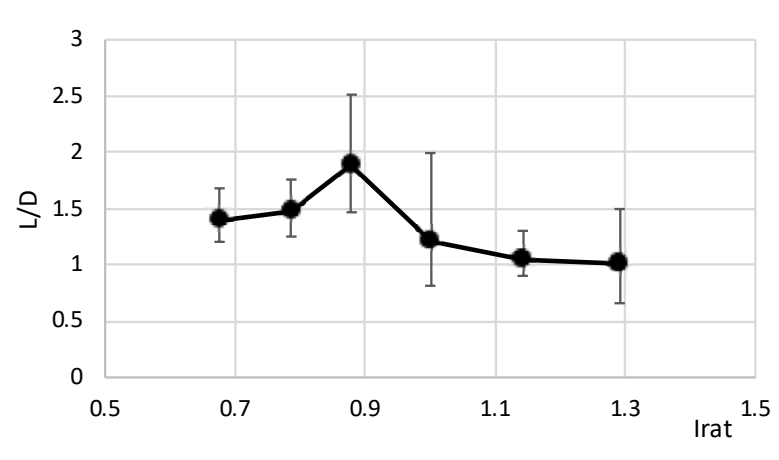

Figure 4: Lift drag ratio.

Directional stability of the models was confirmed that the results are quantitatively the same as listed in [9]. Table 1 shows the percentage of straight trajectory which means that the direction of descending is about to bend by some disturbance, self-correct and descend linearly. Figure 4 and Fig. 5 shows the measured lift drag ratio $L / D$ and velocity in the direction the plate advances due to fall $U_{f f}^{\prime}$ of the plates. The meaning of 'is dimensionless as defined Eq. 37. The error bar is due to the fact that the velocity and aerodynamic force change periodically while the plate rotates, and in addition to it, the movement of the plate changes irregularly by the small disturbance of the surrounding air.

We also acquired data on the effect of shaft length on the trajectory described in 5.1. Where, $I_{r a t}$ means the moment of inertia around the yawing axis ratio written in 4.2.

\section{Wind Tunnel Test}

4.1 Wind Tunnel A vertical type closed return type wind tunnel (Fig. 6) of Aichi Institute of Technology was used for the verification test. The wind tunnel has a test section with dimensions of $0.6 \mathrm{~m}(\mathrm{~W}) \times 0.6 \mathrm{~m}(\mathrm{H}) \times 1.5$ 


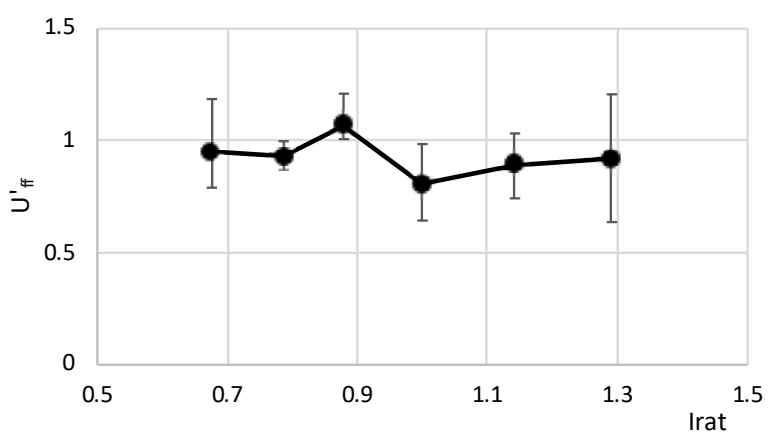

Figure 5: Falling velocity.

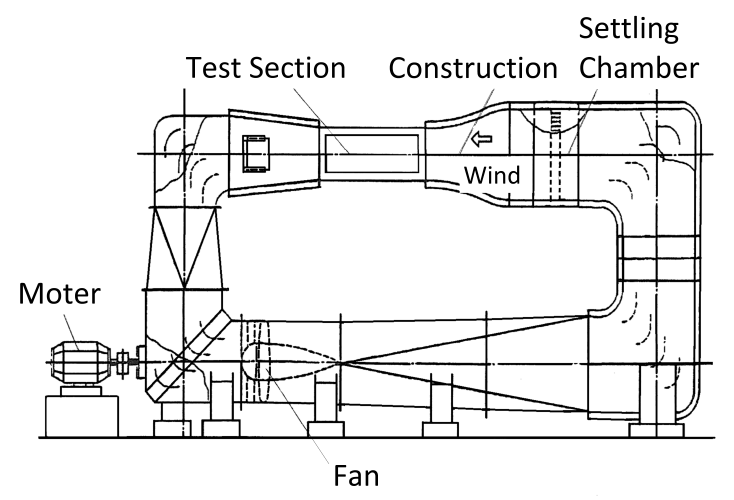

Figure 6: Wind tunnel.

m (L) and can be changed to open type test section by removing the test cart. The maximum wind speed is $50 \mathrm{~m} / \mathrm{s}$.

4.2 Model and Test Equipment The test model is a rectangular plate made of formed Polystyrene sheet, double sided. Configuration parameter of the models are the same as [9],which has a span of $150 \mathrm{~mm}$ and an aspect ratio of 3.75 and is composed of six basic configurations, in which the dents and bulges were parametrically defined by a parabola. Here the meaning of configuration parameter is shown in Table 2. In Table 2, we define $S F$ and $H_{r}$ as following: $S F$ : take the value of $-1.0,-0.5,0.0,0.5,1.0,2.0$ depends on the model shape; $H_{r}: 20 \mathrm{~mm}$ is used in this paper. In addition, $a_{c v}, H_{c e n t e r}$ and $H_{t i p}$ are defined as Eq. 22, Eq. 23 and Eq. 24, respectively.

$$
\begin{aligned}
& a_{c v}=\frac{3 \exp (S F)}{1+2 \exp (S F)} \\
& H_{c e n t e r}=a_{c v} H_{r} \\
& H_{t i p}=H_{r}\left(-2 a_{c v}+3\right)
\end{aligned}
$$

Here we define moment of inertia around the yawing axis ratio to rectangular configuration $I_{r a t}$ as follows,

$$
I_{\text {rat }}=I 2_{S F} / I 2_{S F=0}
$$

where $I 2$ is the moment of inertia around the yawing axis.

$$
I 2=\int_{-b / 2}^{b / 2}\left(C_{\text {center }} y^{2}+\left(C_{\text {tip }}-C_{\text {center }}\right)\left(\frac{2 y}{b}\right)^{2} y^{2}\right) d y
$$

Table 2: Configuration Parameter.

\begin{tabular}{cc}
\hline Config. Parameter & Meaning \\
\hline SF & Shape factor \\
$a_{c v}$ & Convex parameter \\
$H_{r}$ & Half chord length of \\
$H_{\text {center }}$ & rectangular configuration \\
$H_{t i p}$ & Hight at center \\
\hline
\end{tabular}

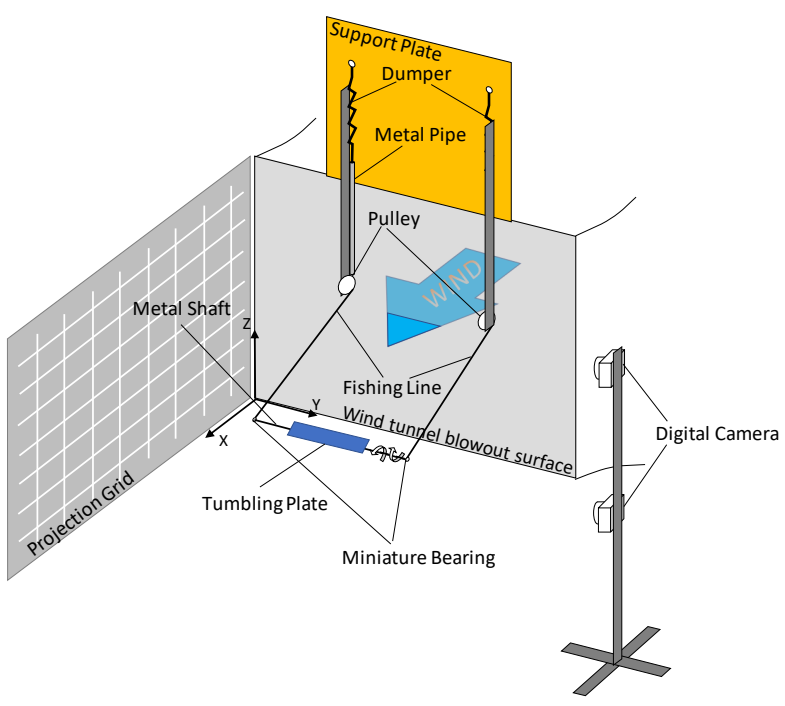

Figure 7: Apparatus setup.

Here, $b$ is span length.

The specifications of the models are shown in Table 3 . In these configurations, the area and span are constant, and the shape of the leading and trailing edges is continuously changed from a convex shape close to a fallen leaf to a conversely concave shape in which the blade tip protrudes centered on a rectangle.

A brass shaft with a diameter of $1 \mathrm{~mm}$ is attached to the plate center line as rotating shaft, the length of the rotating shaft is $164 \mathrm{~mm}$ (the width between the miniature bearings is $160 \mathrm{~mm}$ ) and drawn from the blowout surface of the wind tunnel with with a \#0.6 $(0.12 \mathrm{~mm})$ fishing line through miniature bearing. A setup apparatus of the wind tunnel test is shown in Fig. 7, and a photograph of the test model is shown in Fig. 8. The sideslip angle is set by changing the position of a pulley by shifting it back and forth. Markers are attached to the plate near both ends, and the plate is captured by two cameras set to the test section side surface, projected on the grid wall of the back surface, the coordinates on the grid are read, and three-dimensional coordinates are obtained by using the stereoscopic technique. The fishing line is guided out of the wind tunnel through a metal tube in order to avoid exposure to wind and fixed to the support plate via a rubber damper to suppress vibration.

4.3 Test Conditions Table 4 summarizes the test conditions. The tests were roughly divided into four types which is, (1) shaft length effect test, (2) critical velocity search test, (3) reduced frequency confirmation test and (4) directional stability test. 
Table 3: Specification of the Six Basic Configurations.

\begin{tabular}{cccccccc}
\hline No. & Shape Factor SF & $\mathrm{I}_{\text {rat }}$ & Shape & Span $(\mathrm{mm})$ & Aspect Ration & Thickness $(\mathrm{mm})$ & Weight(g) \\
\hline I & -1 & 1.29 & Concave & & & & \\
II & -0.5 & 1.14 & Concave & & & & \\
III & 0 & 1.00 & Ractangular & 150 & 3.75 & 0.9 & 2.4 \\
IV & 0.5 & 0.88 & Convex & & & & \\
V & 1 & 0.79 & Convex & & & & \\
VI & 2 & 0.68 & Convex & & & \\
\hline
\end{tabular}

Table 4: Test Condition.

\begin{tabular}{|c|c|c|c|c|c|}
\hline Test Item & Model & Velocity & Turning Direction & Ls & $\beta$ \\
\hline Shaft Length Effect & VI & $1.8 \mathrm{~m} / \mathrm{s}$ & Upper/Lower & $\begin{array}{c}50 \mathrm{~mm} \\
30 \mathrm{~mm} \\
15 \mathrm{~mm} \\
7 \mathrm{~mm}\end{array}$ & $0^{\circ}$ \\
\hline Critical Velocity Search & $\begin{array}{c}\text { I } \\
\text { II } \\
\text { III } \\
\text { IV } \\
\text { V } \\
\text { VI }\end{array}$ & Sweep & Upper & $7 \mathrm{~mm}$ & $0^{\circ}$ \\
\hline Reduced Frequency Confirmation & $\begin{array}{c}\text { I } \\
\text { II } \\
\text { III } \\
\text { IV } \\
\text { V } \\
\text { VI }\end{array}$ & $0.7 \sim 2.1 \mathrm{~m} / \mathrm{s}$ & Upper & $7 \mathrm{~mm}$ & $0^{\circ}$ \\
\hline Directional Stability & $\begin{array}{c}\text { I } \\
\text { II } \\
\text { III } \\
\text { IV } \\
\text { V } \\
\text { VI }\end{array}$ & $0.7 \sim 2.1 \mathrm{~m} / \mathrm{s}$ & Upper/Lower & $7 \mathrm{~mm}$ & $0^{\circ},+\Delta$ \\
\hline
\end{tabular}

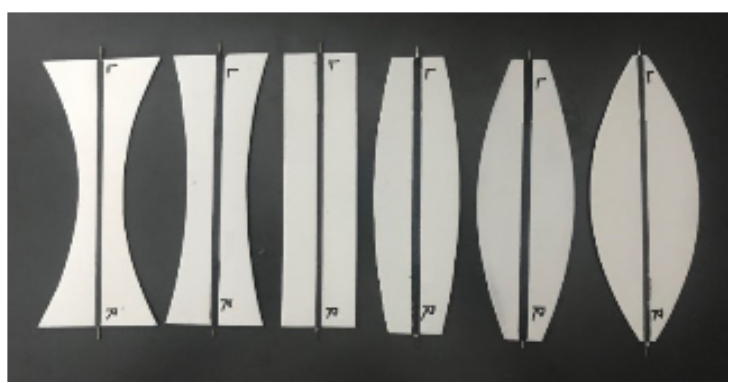

Figure 8: Test models(From left to right, in the order of I, II, III, IV, V, VI).

4.4 Data Processing Flow chart of data processing is shown in Fig. 9 in which general-purpose software used in the process are written.

Slow videos were used to capture images assuming unsteady motion of the tumbling plate. The acquired images are decomposed into frames and combined into one image. From the image data acquired by the two cameras, the twodimensional coordinates projected on the wall surface are read after correcting the distortion of the image and then averaging the unsteady motion, and the three-dimensional

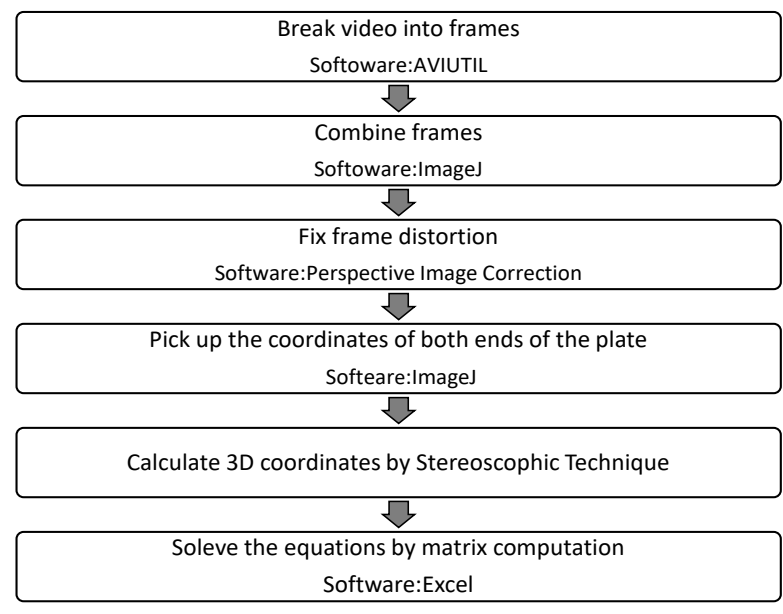

Figure 9: Flow chart.

coordinate data are processed by the method of the general stereoscopic technique using the similarity rule of the triangle as shown in Fig. 10. We put the origin point of the world coordination at the camera center of the lower camera. $P(x, y, z)$ is the three-dimensional position of the point 


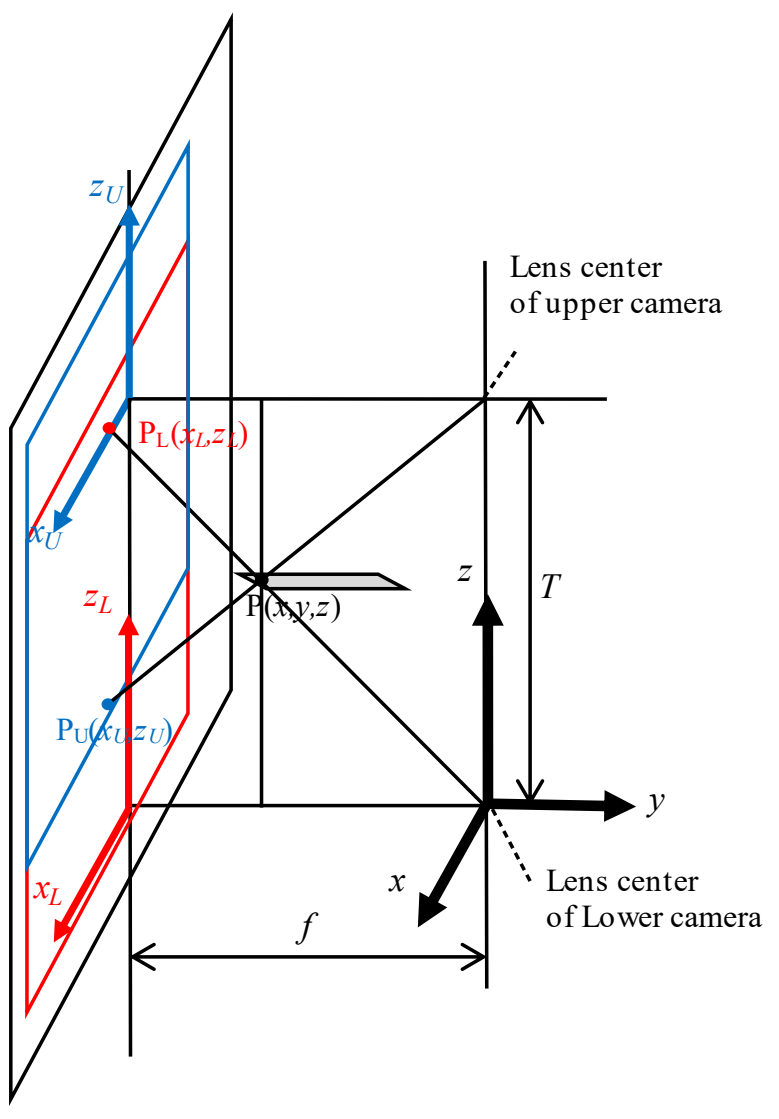

Figure 10: Stereoscopic three-dimensional coordinates.

on the model. $\left(x_{U}, z_{U}\right)$ and $\left(x_{L}, z_{L}\right)$ are point position projected on the aside wall in the upper and lower camera coordination.

The three-dimensional position of the point can be calculated as follows,

$$
\begin{aligned}
& z=\frac{z_{L} T}{z_{L}-z_{U}} \\
& x=\frac{x_{L} T}{z_{L}-z_{U}}=\frac{x_{U} T}{z_{L}-z_{U}} \\
& y=\frac{f T}{z_{L}-z_{U}}
\end{aligned}
$$

\section{Data Accuracy Improvement}

In order to improve the accuracy of the wind tunnel test results, the following effects were examined and corrected.

(1) Effect of the shaft length.

(2) Force applied to the string.

(3) Force applied to the bearing and shaft.

(4) Magnus effect of the shaft.

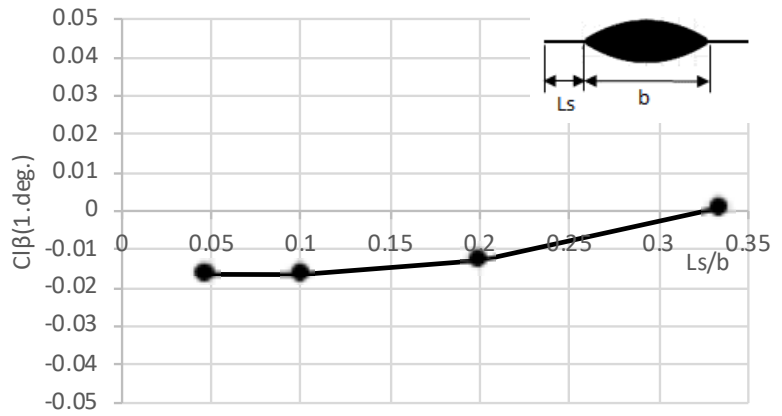

(a) $\mathrm{Cn}_{\beta}$

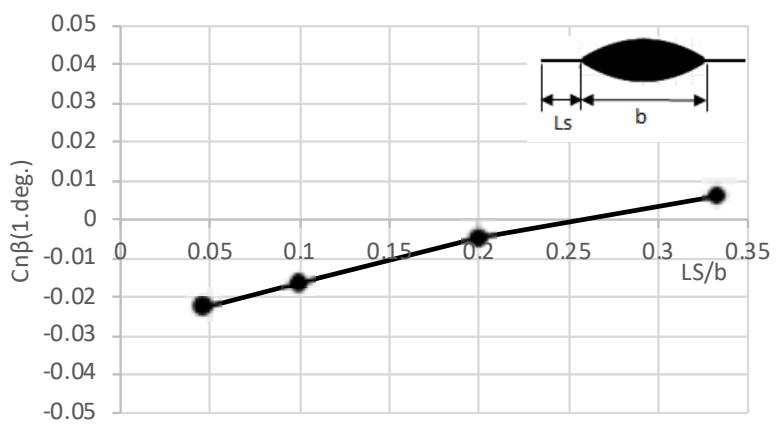

(b) $C l_{\beta}$

Figure 11: Effect of the Shaft Length.

5.1 Effect of the Shaft Length In the preliminary free fall test, it was found that the length of the shaft Ls supporting the model used in this test affects the directional stability. It is considered that this occurs by the unsteady aerodynamic force of the shaft itself affects the directional stability. Figure 11 shows the effect of the shaft length on the directional and lateral stability using configuration VI. Here, $\mathrm{Cn}$ is yawing moment coefficient, $\mathrm{Cl}$ is rolling moment coefficient.

$$
\begin{aligned}
& C l=\frac{l}{1 / 2 \rho U^{2} b^{2} C} \\
& C n=\frac{n}{1 / 2 \rho U^{2} b^{2} C} \\
& C l \beta=\frac{\Delta C l}{\Delta \beta} \\
& C n \beta=\frac{\Delta C n}{\Delta \beta}
\end{aligned}
$$

where, $l$ is rolling moment and $n$ is yawing moment, $U$ is the uniform flow velocity, $C$ is the mean aerodynamic chord and $\rho$ is the air density.

From this result, it can be seen that the shorter the shaft, the better. We decided to set the length of the shaft to $7 \mathrm{~mm}$ $(\mathrm{Ls} / \mathrm{b}=0.047)$, which is the minimum length required to support the rotation of the plate. From the results of Fig. 11, it cannot be said that this length has no effect, but it is considered that there is no problem in evaluating the directional stability. 


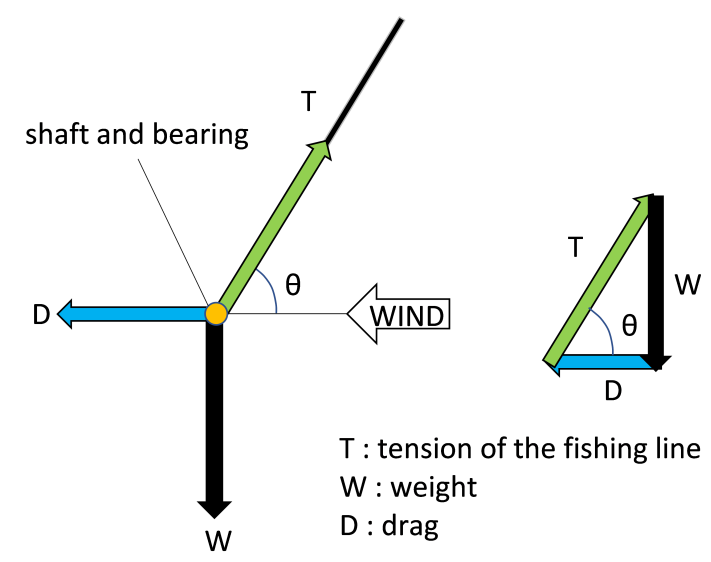

Figure 12: Force balance acing on the shaft and bearing.

5.2 Force Applied to the String Here, the aerodynamic force of the strings exposed to the uniform flow between the miniature bearing and the pulley in Fig. 7 is estimated.

When the diameter of the fishing line is $0.12 \mathrm{~mm}$ and the wind speed is $1.6 \mathrm{~m} / \mathrm{s}$, the Reynolds number Red is $1.3 \times$ $10^{1}$. According to [10], the drag coefficient of a cylinder in this Reynolds number range is about 1.2, and the drag component and lift component when the velocity component acting in the direction perpendicular to the string that looks like this cylinder is tilted by the angle of the string [11]. Think separately for the ingredients. Actually, it is thought that the force acts on the elliptical shape, but by considering it as a cylinder, the case where a larger force acts is calculated.

$$
\begin{aligned}
& C_{\text {Dyaw }}=C_{\text {Dnormal }} \cos ^{2} \Psi \\
& C_{\text {Lyaw }}=C_{\text {Dnormal }} \sin ^{2} \Psi
\end{aligned}
$$

Here, $C_{\text {Dyaw }}$ and $C_{\text {Lyaw }}$ are drag and lift component and $C_{\text {Dnormal }}$ is the drag coefficient when the wind hits the cylinder vertically.

From the experimental results, the angle with the airflow was 0.7 to 0.9 radians for the "upper" rotation and 1.2 to 1.35 radians for the "lower" rotation. From these results, the lift coefficient and drag coefficient acting on the fishing line are calculated as the order of $10^{-4}$, which can be ignored under the test conditions in this paper.

\subsection{Force Applied to the bearings and shafts Here,} the aerodynamic force of the shafts and bearings are estimated and subtracted.

We supported $214 \mathrm{~mm}$ and $164 \mathrm{~mm}$ shafts in a wind tunnel with bearings and fishing lines and measured the amount of movement of the positions at both ends of the shaft with and without wind. Since the shaft and bearing are symmetrical, it seems that only drag is applied and weight of the shaft with bearing is known factor, the drag force can be calculated by measuring the defection angle $\theta$ as Fig. 12 because it is considered that the aerodynamic force applied to the fishing line can be ignored according to the result of the previous section.

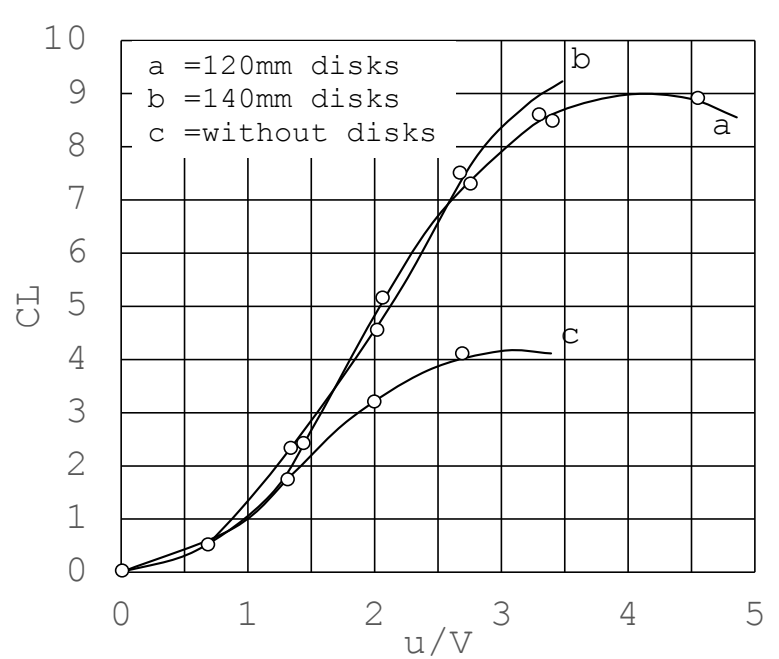

Figure 13: Lift plotted against $u / V .[13]$

Since the difference between the $214 \mathrm{~mm}$ and $164 \mathrm{~mm}$ shaft model drags can be considered as the drag of only the shaft of $50 \mathrm{~mm}$, the drag of the remaining $14 \mathrm{~mm}$ shaft and bearing can be obtained by subtracting the drag acting on only the shaft of $150 \mathrm{~mm}$ from the shaft model of 164 $\mathrm{mm}$. As the result of measurement, $C_{D}$ was calculated as the order of $10^{-3}$. The correction was performed by subtracting this drag from the drag of the model obtained in the wind tunnel test.

5.4 Magnus Effect of the Shaft Magnus effect, which is a force generated in the direction perpendicular to the traveling direction of an object such as a sphere or a cylinder, is well known. In this study, a $1 \mathrm{~mm}$ metal shaft was attached to the tumbling plate. Therefore, the effect of Magnus effect by rotating the shaft itself have to be estimated.

Figure 13 shows the lift coefficient of rotating cylinder from [13]. Here, $u$ means peripheral velocity, $V$ means wind velocity and reference area used to make $C_{L}$ dimensionless is projected area of the cylinder. When reflected on a $1 \mathrm{~mm}$ diameter shaft with a length of $7 \mathrm{~mm}$ on each side, reference area becomes $1.4 \times 10^{-5} \mathrm{~m}^{2}$. Even when the maximum wind speed is $U=2.1 \mathrm{~m} / \mathrm{s}$ under the wind tunnel test conditions in this study, lift force becomes to the order of $10^{-6}$ $\mathrm{N}$ and can be ignored under test condition in this paper.

\section{Test Results}

6.1 Stability due to wind velocity In this towing wind tunnel test method, the model can move freely in the wind tunnel. This can be used to confirm the directional stability of the model. That is, if the directional stability becomes unstable, the model shakes from side to side, and the unstable oscillation occurs. It was confirmed that when the wind speed is gradually increased, oscillation starts when wind speed exceeds a certain critical value. This will be discussed in 6.4. Therefore, when comparing the data of 6 configuration in this study, the wind velocity was set to 1.6 $\mathrm{m} / \mathrm{s}$ where the oscillation does not occur in all configurations. 


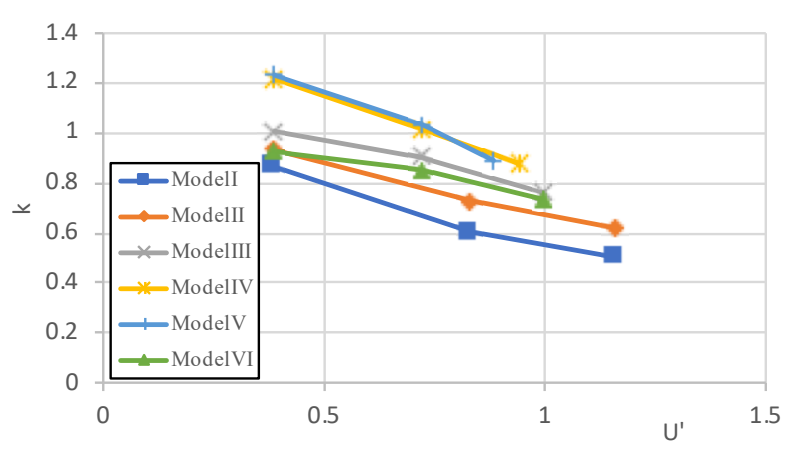

Figure 14: Change in Reduced Frequency $k$.

6.2 Reduced Frequency Figure 14 shows the comparison of reduced frequency $k$ of six models.

For generalization, from Eq. 36 of the balance between gravity and lift force, we make the velocity to dimensionless value as in Eq. 37 using the atmospheric density $\rho$ and surface load $\mathrm{mg} / \mathrm{bC}$ and $C L$ is the lift coefficient defined in Eq. 39.

$$
\begin{aligned}
& m g=\frac{1}{2} \rho U^{2} C_{L} b C \\
& U^{\prime}=U \sqrt{\frac{\rho}{\left(\frac{m g}{b C}\right)}}
\end{aligned}
$$

Definition of $k$ is as follows

$$
k=\frac{\omega C}{2 U}
$$

Where $\omega$ is angler velocity measured in the experiment.

It can be seen that as the velocity increases, the number of revolutions increases, but $k$ itself decreases.

6.3 Lift and Drag Aerodynamic Coefficient Figure 15 shows the lift and drag coefficient by normal definition as,

$$
\begin{aligned}
& C_{L}=\frac{L}{\frac{1}{2} \rho U^{2} b C} \\
& C_{D}=\frac{D}{\frac{1}{2} \rho U^{2} b C}
\end{aligned}
$$

Where, $L$ is the lift force, $D$ is the drag force.

Figure 16 shows the lift drag ratio $L / D$ at $U=1.6 \mathrm{~m} / \mathrm{s}$. Where, $L / D_{w t t}$ means the value from wind tunnel test and $L / D_{f f}$ means the value of free fall test written in chapter 3 . The $L / D$ obtained by wind tunnel test are almost included in error bar of $L / D$ obtained in the preliminary free fall test and can be said to show a good agreement.

\subsection{Lateral and Directional Aerodynamic Coefficient} The authors have reported that the directional stability is affected by the configuration of the tumbling plate, and the falling range of the falling object is depending on the configuration [9]. In this paper, lateral and directional stability of the six configurations tested are investigated.

Figure 17 shows the change in $L / D$ with respect to the wind velocity for model I. From Fig. 14, it can be seen that

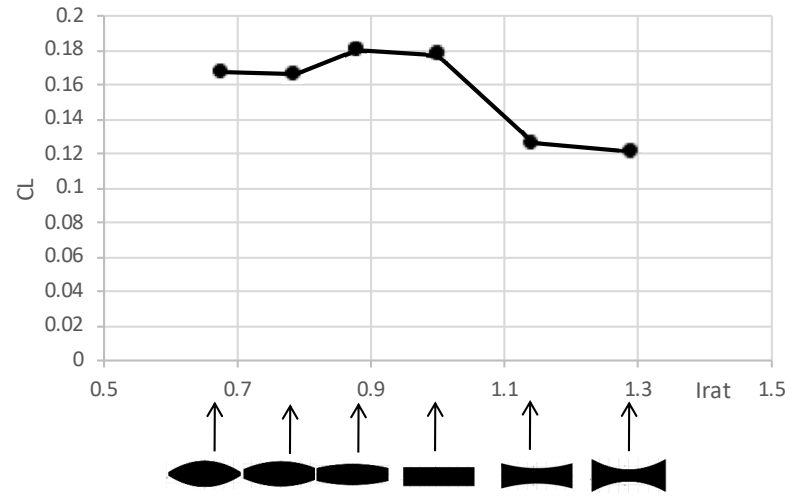

(a) Lift coefficient

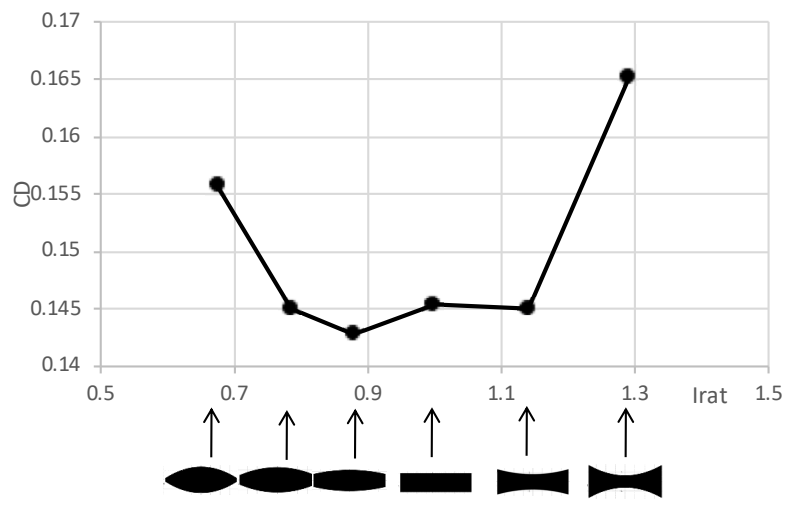

(b) Drag coefficient

Figure 15: Lift and drag coefficient.

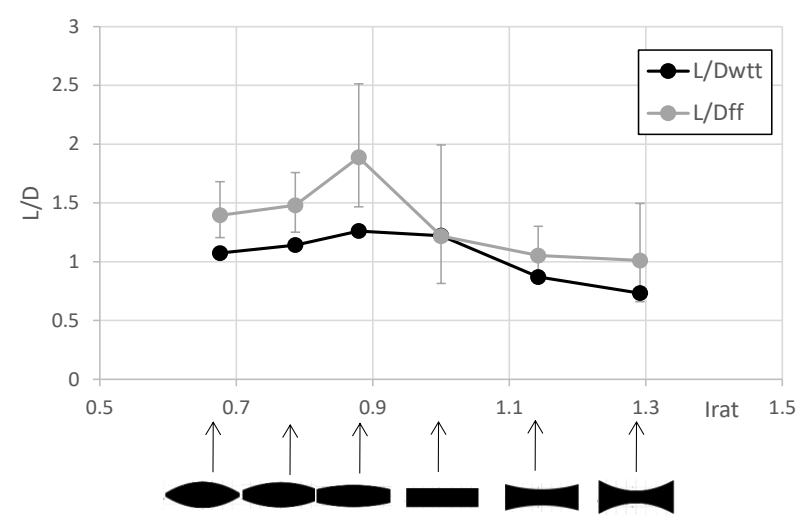

Figure 16: Lift drag ratio.

the reduced frequency tends to decrease as the wind velocity increases, but the $L / D$ hardly changes with the wind velocity. Considering the accidental falling object from the aircraft, it is considered that when the shape is the same, the falling speed increases with the increase in the weight of the falling object from the balance in the air, but the $L / D$ hardly changes. It can be seen that the effect on the reach distance is small.

Figure 18 shows the Critical Velocity $U c$ in which the model starts to oscillate when $\beta$ is set to $0^{\circ}$ and the wind velocity is gradually increased. For Model I, oscillation did not appearat wind speeds up to $U^{\prime}=2.76(5.0 \mathrm{~m} / \mathrm{s})$ conducted in this test, and it did not become unstable. 


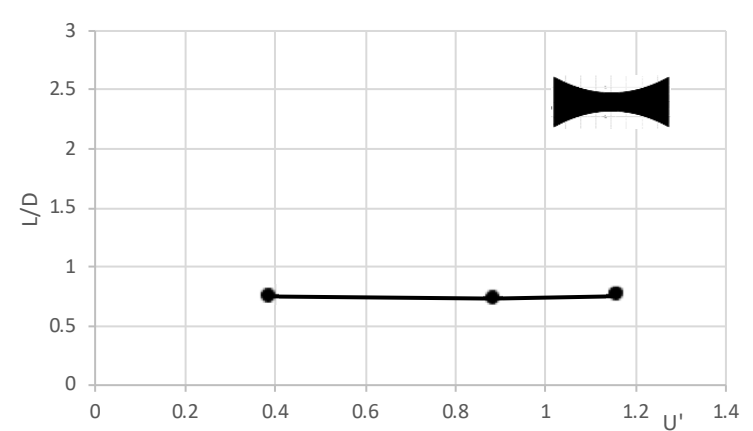

Figure 17: Change in $L / D$.

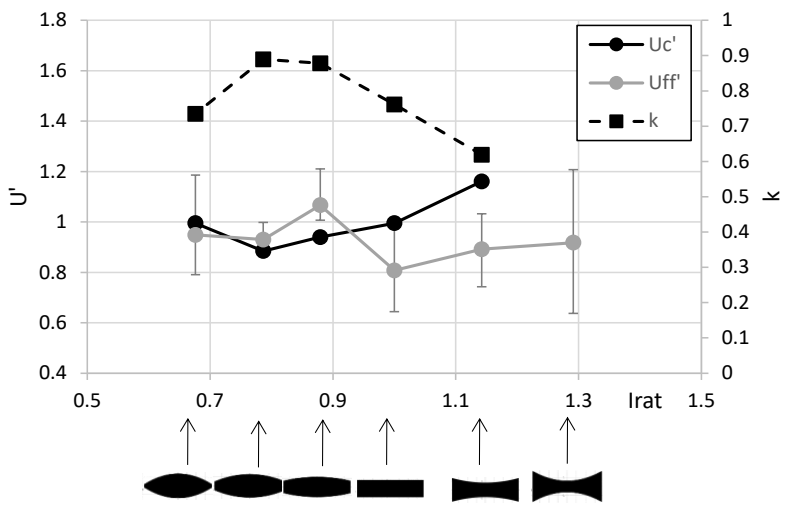

Figure 18: Critical Velocity.

Figure 18 also superimposes the falling velocity obtained in the Free Fall Test. From this, it can be seen that the directional stability shown in Table 1 is determined by the relationship with the falling velocity for each configuration. That is, it is considered that models I and II fall in a sufficiently stable speed range, but models IV to VI fall at a speed exceeding $U c^{\prime}$, causing directional instability and started turning. In addition, it was found that for model III, directional instability may appear depending on the state of the fluctuation range because $U c^{\prime}$ exists at the upper limit of the speed fluctuation range of the fall.

As an indicator of vertical and horizontal stability, $\mathrm{Cn} \beta$ and $C l \beta$ at $U^{\prime}=1.00(1.8 \mathrm{~m} / \mathrm{s})$ is shown in Fig. 19 and Fig. 20. As shown in Fig. 18, this speed is a speed range in which stability and instability are divided depending on the configuration of the model. At lower speeds, all models are stable, and at high speeds, oscillation appears, so force measurement is not possible. At this wind velocity, Model IV and V have already oscillated and cannot be measured. Regarding directional stability $C n \beta$, Models I and II are stable, and III and VI are unstable, which agree with the results in Table 1. On the other hand, regarding the lateral stability $C l \beta$, it is negative in all configurations, indicating that it is stable, but this means that if a sideslip angle occurs due to some disturbance, a turn is started to correct the sideslip. So, it is considered that it does not contradict the results in Table 1.

6.5 Unsteady Motion Figure 21 shows the unsteady typical tip position $X$ of model I and VI when $U^{\prime}=0.88(1.6$ $\mathrm{m} / \mathrm{s}$ ) and $\beta=0^{\circ}$. There is no noticeable difference leading to

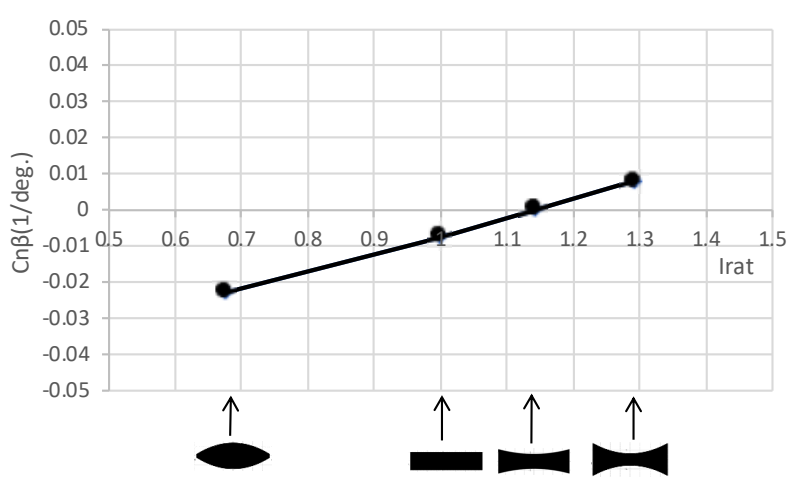

Figure 19: $C n_{\beta}$.

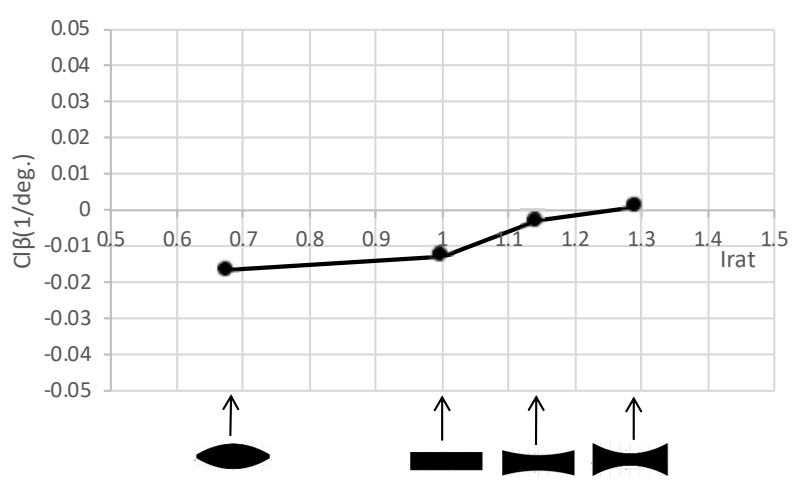

Figure 20: $C l_{\beta}$.

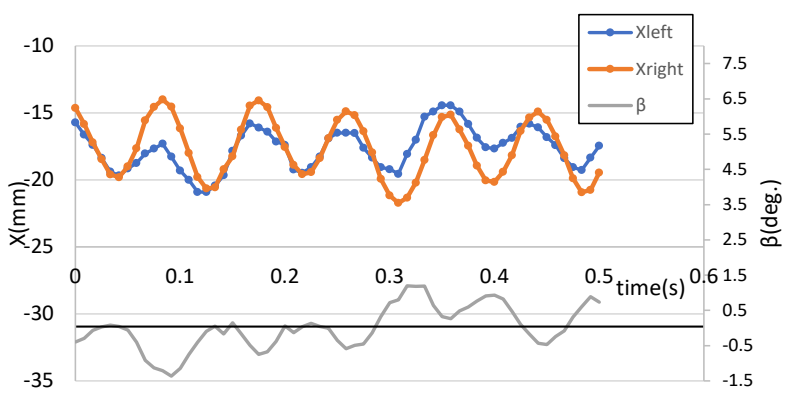

(a) Model I

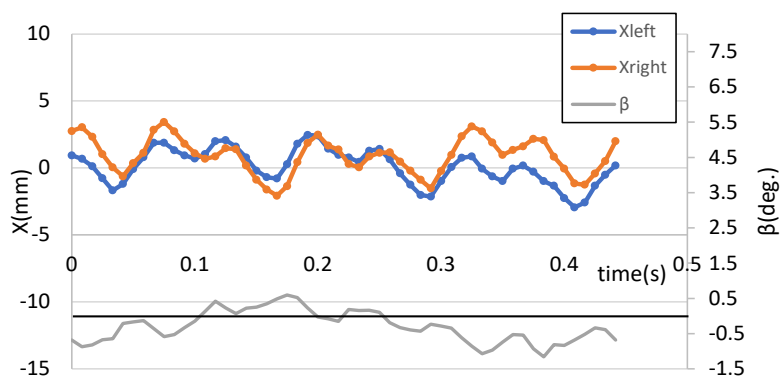

(b) Model VI

Figure 21: Unsteady Tip Position Movement.

directional instability in the movement of the left and right tips, but model I has a stable periodic movement, but VI has irregular movements, and it can be imagined that it is a sign of instability. 


\section{Conclusion}

In order to investigate the directional stability of the basic configuration, the force was measured in a wind tunnel test using the towing wind tunnel test method. As a result, the following became clear.

(a) When the wind velocity is gradually increased, there exists a critical velocity in which the model becomes unstable and begins to oscillate laterally, which depends on the configuration.

(b) The $L / D$ obtained by wind tunnel test are almost included in error bar of $L / D$ obtained in the preliminary free fall test and can be said to show a good agreement.

(c) Although the reduced frequency tends to decrease as the wind speed increases, but the $L / D$ hardly changes with the wind speed. This leads to the fact that even if the weight of the falling object increases and the falling speed increases, the influence on the reach distance is considered small.

(d) The critical velocity written (a) consistent with the directional instability phenomena due to the configuration of the free fall test. That is, it was confirmed that the configuration considered to be directionally unstable in the free fall test was caused by the falling speed exceeding the critical speed.

Since the unsteady aerodynamic force cannot be measured by the towing wind tunnel test method used in this study, the unsteady aerodynamic force that causes directional instability has not been measured. In the future, it is hoped that the phenomenon of unstable aerodynamic force generation due to configuration will be investigated by measuring unsteady aerodynamic force by some method.

\section{References}

[1] B. Kakimpa, D. M. Hargreaves and J. S. Owen, "An investigation of plate-type windborne debris flight using coupled CFD-RBD models. Part I: Model development and validation", Journal of Wind Engineering and Industrial Aerodynamics, Vol.111, pp.95-103, 2012. DOI: 10.1016/j.jweia.2012.07.008

[2] B. Kakimpa, D. M. Hargreaves and J. S. Owen, "An investigation of plate-type windborne debris flight using coupled CFD-RBD models. Part II: Free and constrained flight", Journal of Wind Engineering and Industrial Aerodynamics, Vol.111, pp.104-116, 2012. DOI: 10.1016/j.jweia.2012.07.011

[3] D. M. Hargreaves, B. Kakimpa and J. S. Owen, "The computational fluid dynamics modeling of the autorotation of square, flat plates", Journal of Fluids and Structures, Vol.46, pp.111-133, 2014. DOI: 10.1016/j.jfluidstructs.2013.12.006

[4] Y. Tanabe and K. Kaneko, "Behavior of a Falling Paper”, Physical Review Letters, Vol.73, No.10, pp.1372-1375, 1994. DOI: 10.1103/PhysRevLett.73.1372
[5] A. C. DeVoria and K. Mohseni, "A vortex model for forces and moments on low-aspect-ratio wings in side-slip with experimental validation", Proceedings of the royal society A, Vol.473, No.2198, 2017. DOI: 10.1098/rspa.2016.0760

[6] A. Andersen, U. Pesavento and Z. J. Wang, "Unsteady aerodynamics of fluttering and tumbling plates", Journal of Fluid Mechanics, Vol.541, pp.65-90, 2005. DOI: 10.1017/S002211200500594X

[7] W. B. Wang, R. F. Hu, S. J. Hu and Z. N. Wu, "Influence of aspect ratio on tumbling plates", Journal of Fluid Mechanics, Vol.733, pp.650-679, 2013. DOI: 10.1017/jfm.2013.461

[8] K. Hirata, K. Shimizu, K. Fukuhara, K. Yamauchi, D. Kawaguchi and J. Funaki, "Aerodynamic Characteristics of a Tumbling Plate under Free Flight", Journal of Fluid Science and Technology, Vol.4, No.1, pp.168-187, 2009. DOI: $10.1299 /$ jfst. 4.168

[9] M. Ishiguro and Y. Miyake, "Experimental Study on Directional Stability of Tumbling Plate", Journal of the Institute of Industrial Applications Engineers, Vol.9, No.1, pp.1-8, 2021. DOI: 10.12792/JIIAE.9.1

[10] S. F. Hoerner, "Fluid dynamic drag", published by the author, 1958. DOI: $10.1017 / \mathrm{s} 0368393100069200$

[11] J. C. Stroman, "Aerodynamic drag coefficients of a variety of electrical conductors", A thesis in Mechanical Engineering, Texas Tech University, 1997.

[12] M. Ishiguro and Y. Miyake, "Proposal of a method to measure aerodynamic forces without using force sensors in wind tunnel testing of tumbling plates", Journal of the Institute of Industrial Applications Engineers, Vol.9, No.3, pp.75-84, 2021. DOI: 10.12792/JIIAE.9.75

[13] J. Ackeret, "Recent experiments at the Gottingen aerodynamic institute", Technical Report Archive \& Image Library, 1925.

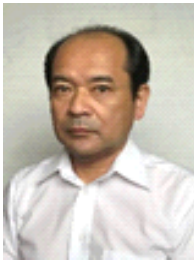

Mitsuo Ishiguro (Member) was born in Aichi, Japan, on May 6, 1961. He received a Ph.D. degree in engineers from Nagoya University in 1995, and is presently Part-time lecturer of Aichi Institute of Technology.

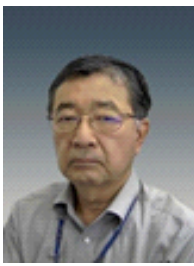

Yoshiaki Miyake (Non-member) was born in Kyoto, Japan, on July 15, 1955. He received a Dr. degree in engineering from Kyoto University in 2009, and is presently a Professor at the Department of Mechanical Engineering, Aichi Institute of Technology. 